\title{
Traumatic hyphema in a 13-year-old girl: eye protection regulation in badminton is needed
}

\author{
Dan Ni Wang BS, Micah Luong MD, Christopher Hanson MD
}

Cite as: CMAJ 2020 July 6;192:E778-80. doi: 10.1503/cmaj.191273

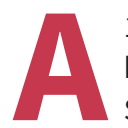

13-year-old girl was assessed at our eye care clinic after being struck in the left eye by a badminton shuttlecock. She was not wearing protective eyewear at the time of injury. Visual acuity in the patient's left eye was hand motion and her intraocular pressure (IOP) was 19 (normal 11-21) $\mathrm{mm} \mathrm{Hg}$. Slit-lamp examination showed a thick clot of hemorrhage in the anterior chamber (Figure 1). We diagnosed a traumatic hyphema and managed the patient with topical prednisolone acetate and atropine $1 \%$ drops, an eye shield and bed rest. We also advised that she sleep with the head of the bed elevated and return to the clinic if her vision decreased.

At the 1-week follow-up visit, the patient showed substantial improvement in visual acuity to $20 / 70$ in the left eye.

\section{Discussion}

An estimated 3.7\% of Canadians older than 15 years, and 2.7\% between age 5 and 14 years, play badminton. ${ }^{1}$ It is a popular sport among youth and adults and is generally considered to have a low risk of injury. However, badminton is the leading cause of racquet sports-related eye injuries in Canada. ${ }^{2}$ The overall incidence of sports-related eye injuries in Canada in 2012 was $8.6 \%$, with the most common causes being hockey, racquet

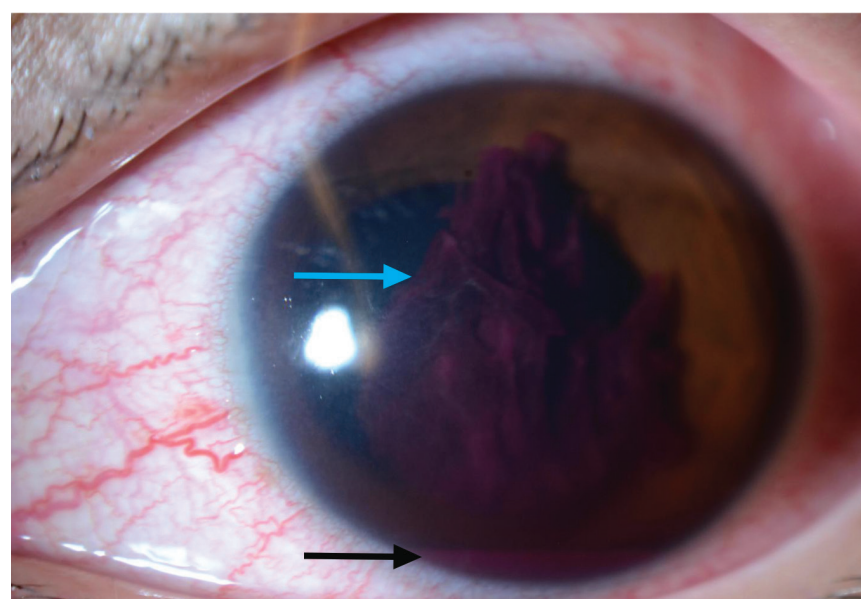

Figure 1: Slit-lamp photography of the left eye of a 13-year-old girl, showing a 1-mm inferior hyphema (black arrow) and clot in the anterior chamber partially obstructing the iris (blue arrow).

\section{KEY POINTS}

- Badminton is the leading cause of racquet sports-related eye injuries in Canada, and traumatic hyphema is a common complication.

- More than half of patients with ocular injuries from badminton never regain full visual acuity.

- Ocular injury from badminton can be prevented with the use of eye protectors that meet standard F803 of the American Society for Testing and Materials.

sports and baseball. ${ }^{3}$ Most eye injuries from badminton occur from shuttlecock impact, because of its small base diameter; high velocity, which can reach more than $482 \mathrm{~km} / \mathrm{h}(137 \mathrm{~m} / \mathrm{s})$; and the relatively close distance between players during games. ${ }^{4}$ The majority of ocular injuries in badminton occur during doubles games, where injury can be caused by an opponent's shuttlecock, the racquet of a partner or a player looking back toward a partner during a "smash" hit. ${ }^{5}$

Blunt trauma to the eye from sports can result in a wide variety of presentations. Traumatic hyphema is the most common ocular presentation from eye injury in badminton, with the majority of cases occurring in children. ${ }^{5}$ Traumatic hyphema occurs when blood pools in the anterior chamber after blunt force to the orbit causes rupture of the vessels that supply the iris and ciliary body. ${ }^{3}$ Patients typically present with blurred vision and pain after blunt eye trauma; severity can range from a microhyphema to a total or complete hyphema. Examination of the eye will show fresh or clotted blood that pools in the anterior chamber. Initial evaluation includes a thorough history and physical examination of the eye, including testing for visual acuity, IOP, extraocular movements, slit-lamp examination and a dilated fundus examination. Patients should be referred immediately to an ophthalmologist for a comprehensive assessment to rule out other associated eye injuries, and receive close follow-up to monitor for complications if a hyphema is suspected.

Initial management of a hyphema includes conservative measures to promote resorption of blood, including bed rest, elevation of the head of the bed at night and an eye shield. ${ }^{6} \mathrm{~A}$ topical cycloplegic such as atropine and a corticosteroid eye 
drop may be applied, although evidence on efficacy is weak. ${ }^{6} \mathrm{~A}$ recent Cochrane review found limited evidence for the use of antifibrinolytics, including tranexamic acid and aminocaproic acid. ${ }^{7}$ Acetylsalicylic acid and nonsteroidal anti-inflammatories should be avoided as they can increase the risk of further bleeding. Elevated IOP is common, owing to occlusion of the trabecular meshwork by the clot or inflammatory cells, and can be controlled with topical $\beta$-blocker drops. Patients should be advised to return to the clinic or to the emergency department immediately if they experience a further worsening in vision, as it may indicate a rebleed.

The long-term sequelae of a hyphema can vary. A secondary hemorrhage, or rebleed, occurs in about $5 \%$ of cases and is associated with worse visual outcomes. ${ }^{6}$ It most often presents between the first 2 and 5 days after injury as a new bleed that is often of greater magnitude than the initial hyphema and is associated with vision-threatening complications, including corneal bloodstaining, secondary glaucoma and optic nerve damage. Predictors of a rebleed include delayed medical assessment for longer than 24 hours after the injury, a larger initial hyphema, elevated IOP $>21 \mathrm{~mm} \mathrm{Hg}$ and initial visual acuity worse than 20/200. ${ }^{6}$ Patients with sickle cell trait or disease also have a higher incidence of rebleed and glaucoma. Surgery may be indicated in some severe and nonresolving cases. Hyphemas can be managed safely in an outpatient setting but hospital admission is indicated for patients who are at risk of noncompliance with bed rest, have severe associated ocular trauma, or have sickle cell disease. Secondary glaucoma, optic neuropathy and early cataract formation are common and can present anywhere from days to years after injury. Therefore, annual follow-up is necessary after resolution.

Permanent vision loss is a devastating consequence of sportsrelated eye injuries. Chandran and colleagues looked at 63 cases of unilateral ocular injury from badminton between 1968 and 1972 in Malaysia; most patients presented with hyphema, and more than half had some degree of chronic vision loss. ${ }^{5}$ Kelly and colleagues looked at 6 cases of badminton eye injuries in competitive players treated at Leicester Royal Infirmary in Manchester between 1979 and 1985, of which 2 players suffered permanent vision loss. ${ }^{8}$ All studies noted that many of those injured were not wearing protective eyewear.

Mandatory eye protection is currently required across many sports internationally, including baseball, hockey, football, lacrosse and squash. ${ }^{9}$ Badminton remains one of the only highrisk sports without formal eye protection regulations. ${ }^{9}$ Ocular injuries from sports affect all age groups and levels of players, and more than $90 \%$ are preventable with protective eyewear. ${ }^{10}$ Previously, squash and racquetball accounted for $73 \%$ of sportsrelated eye injuries. The implementation of mandatory eye protection in squash and racquetball in 1982 caused this proportion to decrease from $73 \%$ to $23 \%$ by $1993 .{ }^{10}$ Similar declines would be expected if implemented in badminton.

Canadian regulations and recommendations for protective eyewear are shown in Box 1. In 2005, the Ontario Badminton Association mandated that all junior double players in competitive games wear protective eyewear that meet the international American Society for Testing and Materials (ASTM) F803 standard. British Columbia is the only other province that has mandated F803 eyewear for junior players younger than 19 years. Newfoundland and Labrador mandates protective eyewear for junior girls in mixed-doubles competitions, Prince Edward Island requires junior players to wear eyewear for doubles and mixed-doubles competitions, and Nova Scotia requires players younger than 19 years to wear goggles for doubles and mixed doubles. Alberta, Quebec and Saskatchewan informally recommend the use of polycarbonate protective eyewear. The

Box 1: Formal regulations and recommendations for protective eyewear, by province or territory

Province/territory

Alberta

British Columbia

Manitoba

New Brunswick

Newfoundland and Labrador

Northwest Territories

Nova Scotia

Nunavut

Ontario

Prince Edward Island

Quebec

Saskatchewan

Yukon
Formal regulation and recommendation for protective eyewear

No formal regulations; informally recommends the use of polycarbonate protective eyewear. Mandated ASTM F803 eyewear for junior players younger than 19 years.

No formal regulations or recommendations.

No formal regulations or recommendations.

Mandates protective eyewear for junior girls in mixed-doubles competitions.

No formal regulations or recommendations.

Requires players younger than 19 years to wear goggles for doubles and mixed-doubles games.

No formal regulations or recommendations.

In 2005, the Ontario Badminton Association mandated that all junior double players in competitive games wear protective eyewear that meets the international technical ASTM F803 standard.

Requires junior players to wear eyewear for doubles and mixed-doubles competitions.

No formal regulations; informally recommends the use of polycarbonate protective eyewear.

No formal regulations; informally recommends the use of polycarbonate protective eyewear.

No formal regulations or recommendations.

Note: ASTM = American Society for Testing and Materials. 
remaining provinces and territories have no recommendations, to the best of our knowledge. Individual school boards, including those of Ontario and Saskatchewan, require F803 protective eyewear for all students.

Only eye guards that meet the ASTM technical standard should be used in racquet sports, because they are made from durable polycarbonate plastic and protect from impact on all sides. ASTM F803-certified eyewear is labelled with a Canadian Standards Association logo for easy identification and is available at most sporting goods stores. Noncertified eye guards and prescription eyeglasses should not be worn, because of the risk of shattering the lens and causing open globe injury. ${ }^{7}$

The risk of permanent eye injury from badminton corroborates the need for increased awareness and protective eyewear regulations. Players report discomfort, cost and low perception of risk of injury as reasons for not wearing protective eyewear during games. However, increased education and awareness regarding the risk of eye injury in badminton may help players to make informed choices about wearing eye protection while playing.

This case highlights a presentation of ocular trauma resulting from badminton that was preventable with eye protection. In consideration of the risk of ocular injury and potential long-term complications when playing badminton, we encourage greater awareness of the importance of the use of eye protection in the game. Furthermore, we encourage Canadian badminton associations to consider adopting regulations requiring ASTM F803 standardapproved protective eyewear on court.

\section{References}

1. Most practiced sports by Canadians, 2005. Ottawa: Statistics Canada; modified 2011 Aug. 30. Available: https://www150.statcan.gc.ca/n1/pub/81-595-m /2008060/t-c-g/tbl6-eng.htm (accessed 2019 Dec. 7).

2. Pashby TJ. Eye injuries in Canadian sports and recreational activities. Can J Ophthalmol 1992;27:226-9.

3. Gordon KD. The incidence of eye injuries in Canada. Can J Ophthalmol 2012;47:351-3.

4. Cohen C, Texier B, Quéré D, et al. The physics of badminton. New J Phys 2015; 17:063001.

5. Chandran S. Ocular hazards of playing badminton. Br J Ophthalmol 1974;58:757-60.

6. Walton W, Von Hagen S, Grigorian R, et al. Management of traumatic hyphema. Surv Ophthalmol 2002;47:297-334.

7. Gharaibeh A, Savage HI, Scherer RW, et al. Medical interventions for traumatic hyphema. Cochrane Database Syst Rev 2019;(1):CD005431.

8. Kelly SP. Serious eye injury in badminton players. Br J Ophthalmol 1987;71:746-7.

9. Dain SJ. Sports eyewear protective standards. Clin Exp Optom 2016;99:4-23.

10. Goldstein MH, Wee D. Sports injuries: an ounce of prevention and a pound of cure. Eye Contact Lens 2011;37:160-3.

Competing interests: None declared.

This article has been peer reviewed.

The authors have obtained consent from the patient's family.

Affiliations: Cumming School of Medicine (Wang) and the Division of Ophthalmology (Luong, Hanson), University of Calgary, Calgary, Alta.

Contributors: All of the authors contributed to the conception and design of the work, drafted the manuscript, revised it critically for important intellectual content, gave final approval of the version to be published and agreed to be accountable for all aspects of the work.

Correspondence to: Dan Ni Wang, danni.wang1@ucalgary.ca 